\title{
Synthesis, Characterization and Antimicrobial Activity of Some Newly Synthesized 1,3-Thiazolidin-4-one Derivatives
}

\author{
ARJUNSINGH K.RANA, SUNIL B. LADE, MAYUR J. JOSHI and NIRAV M. SHAH* \\ Department of Chemistry, Navjivan Science College, Dahod-389 151, Gujarat, India \\ *Oxygen Healthcare Limited, Ahmedabad, India \\ nirav.shah.m@gmail.com
}

Received 14 July 2012 / Accepted 16 August 2012

\begin{abstract}
A series of 10 new 1,3-thiazolidin-4-one (3a-3j) were synthesized from different schiff base of $N$-[(1E)-(3-chloro-5,7-dimethyl-2-naphthyl) methylene]aniline (2a-2j) with thioaceticacid. The chemical structures of these compounds were confirmed by means of ${ }^{1} \mathrm{H}$ NMR, IR and mass analysis. Newly synthesized compounds were screened in vitro for their antimicrobial activity against varieties of bacterial strains such Staphylococcus epidermidis, Staphylococcus aureus, Escherichia coli, Pseudomonas aeruginosa and fungi strain Aspergillus niger at $40 \mu \mathrm{g} / \mathrm{mL}$. The resulted of compounds shows that chloro and flouro substituted 1,3-thiazolidine-4-one derivatives are showing quite well activity as compare to the other functional groups.
\end{abstract}

Keywords : 1,3-Thiazolidin-4-one, 2-Chloro-6, 8-dimethyl quinoline-3-carboxaldehyde, Schiff base, Antimicrobial evaluation

\section{Introduction}

The massive use of antibacterial drugs by mankind leads to a major problem i.e. drug resistance. The emergence of the drug resistance for major classes of antibacterial drugs is recognized by WHO and this became challenge for medicinal chemist to develop new class of antimicrobial agents. With increasing number of cases of pathogenic infection which suppress the immune system of human, medicinal chemist decided to move towards synthesis of small and novel molecule.

Quinoline nucleus has received remarkable attention in medicinal science. Quinoline has prominent structural feature in a variety of natural products as well as in other compounds of medicinal interest. Quinoline derivatives are reported to possess anticonvulsant, antitumor ${ }^{1}$, hypnotics, sedative, antihypertensive, antimalarial ${ }^{2}$, antibacterial ${ }^{3}$ and many other therapeutic activities. The 4-thiazolidinone ring system exhibit various biological activities, such as antimicrobial, anti-inflammatory, anti-HIV, anti-toxoplasma gondii and analgesic ${ }^{4-8}$.

From literature survey we have identified that 2-chloro-6,8-dimethyl quinoline-3carbaldehyde and its derivatives are associated with many diverse therapeutic activity hence it is worthwhile to select as nucleus. 


\section{Experimental}

The melting points were determined in open capillary tubes and are uncorrected. IR spectra were recorded on Shimadzu FT-IR-8400 spectrophotometer using $\mathrm{KBr}$ disc and ${ }^{1} \mathrm{H}$ NMR spectra in DMSO- $\mathrm{d}_{6}$ (Chemical shift in $\left.\delta \mathrm{ppm}\right)$ on Bruker spectrometer $(400 \mathrm{MHz})$ using TMS as an internal standard. The results are in good agreement with the structure assigned. The purity of all compounds was checked by thin layer chromatography using TLC plates of silica gel (E.Merck G254) using Ethyl acetate: Hexane solvent system (7:3). Physical Constants synthesized of compounds (3a-3j) are recorded in Table 1.

Table 1. Physical constants 2-(3-chloro-5,7-dimethyl-2-naphthyl)-3-substituted phenyl-1,3thiazolidin-4-one (3a-3j)

\begin{tabular}{|c|c|c|c|c|c|c|c|}
\hline $\begin{array}{c}\text { S } \\
\text { No. }\end{array}$ & Compd. & $\mathrm{R}$ & $\begin{array}{l}\text { Molecular } \\
\text { Formula }\end{array}$ & $\begin{array}{l}\text { Mol. } \\
\text { Wt }\end{array}$ & Yield & $\underset{{ }^{0} \mathrm{C}}{\mathrm{M} . \mathrm{P}}$ & $\mathrm{R}_{\mathrm{f}}$ \\
\hline 1 & 3a & $\mathrm{C}_{6} \mathrm{H}_{5^{-}}$ & $\mathrm{C}_{20} \mathrm{H}_{17} \mathrm{ClN}_{2} \mathrm{OS}$ & 369 & $78 \%$ & 167 & 0.54 \\
\hline 2 & $3 \mathbf{b}$ & 4- $\mathrm{Br} \mathrm{C}_{6} \mathrm{H}_{4^{-}}$ & $\mathrm{C}_{20} \mathrm{H}_{16} \mathrm{BrClN}_{2} \mathrm{OS}$ & 448 & $68 \%$ & 177 & 0.55 \\
\hline 3 & $3 c$ & $4-\mathrm{Cl}-\mathrm{C}_{6} \mathrm{H}_{4}^{-}$ & $\mathrm{C}_{20} \mathrm{H}_{16} \mathrm{Cl}_{2} \mathrm{~N}_{2} \mathrm{OS}$ & 403 & $65 \%$ & 182 & 0.57 \\
\hline 4 & 3d & 2,4-di Cl- ${ }_{6} \mathrm{H}_{3}$ & $\mathrm{C}_{20} \mathrm{H}_{15} \mathrm{Cl}_{3} \mathrm{~N}_{2} \mathrm{OS}$ & 437 & $74 \%$ & 189 & 0.53 \\
\hline 5 & $3 \mathbf{e}$ & $4-\mathrm{F}-\mathrm{C}_{6} \mathrm{H}_{4^{-}}$ & $\mathrm{C}_{20} \mathrm{H}_{16} \mathrm{ClFN}_{2} \mathrm{OS}$ & 387 & $78 \%$ & 190 & 0.68 \\
\hline 6 & $3 f$ & $4-\mathrm{NO}_{2}-\mathrm{C}_{6} \mathrm{H}_{4-}$ & $\mathrm{C}_{20} \mathrm{H}_{16} \mathrm{ClN}_{3} \mathrm{O}_{3} \mathrm{~S}$ & 414 & $70 \%$ & 175 & 0.74 \\
\hline 7 & $3 g$ & $4-\mathrm{SCH}_{3}-\mathrm{C}_{6} \mathrm{H}_{4}^{-}$ & $\mathrm{C}_{21} \mathrm{H}_{19} \mathrm{ClN}_{2} \mathrm{OS}_{2}$ & 415 & $62 \%$ & 172 & 0.48 \\
\hline 8 & 3h & $4-\mathrm{OCH}_{3}-\mathrm{C}_{6} \mathrm{H}_{4}-$ & $\mathrm{C}_{21} \mathrm{H}_{19} \mathrm{ClN}_{2} \mathrm{O}_{2} \mathrm{~S}$ & 399 & $81 \%$ & 182 & 0.32 \\
\hline 9 & $3 \mathbf{i}$ & $4-\mathrm{CH}_{3}-\mathrm{C}_{6} \mathrm{H}_{4}^{-}$ & $\mathrm{C}_{21} \mathrm{H}_{19} \mathrm{ClN}_{2} \mathrm{OS}$ & 382 & $75 \%$ & 197 & 0.36 \\
\hline 10 & $3 \mathbf{j}$ & 2,4-di $\mathrm{CH}_{3}-\mathrm{C}_{6} \mathrm{H}_{3}-$ & $\mathrm{C}_{22} \mathrm{H}_{21} \mathrm{ClN}_{2} \mathrm{OS}$ & 397 & $70 \%$ & 163 & 0.57 \\
\hline
\end{tabular}

General procedure for synthesis of $N$-[(1E)-(3-chloro-5,7-dimethyl-2-naphthyl) methylene] aniline (2a-2j)

To a stirred solution of 2-chloro-6, 8-dimethyl quinoline-3-carboxaldehyde (0.01 mol) in ethanol, substituted aniline $(0.01 \mathrm{~mol})$ and catalytic amount of acetic acid $(0.0005 \mathrm{~mol})$ was added. The mixture was refluxed for 6 hours. The contents were cooled and the filtered the isolated product. Product was crystallized from appropriate solvent.

General procedure for synthesis 2-(3-chloro-5,7-dimethyl-2-naphthyl)-3-substituted phenyl-1,3-thiazolidin-4-one (3a-3j)

To a stirred solution of $\mathbf{2 a - 2} \mathbf{j}(0.01 \mathrm{~mol})$ in dioxan, thioglycolic acid $\left(\mathrm{SH}-\mathrm{CH}_{2}-\mathrm{COOH}\right)$ $(0.01 \mathrm{~mol})$ was added and refluxed the reaction mass for $12 \mathrm{~h}$. The reaction mixture was cooled and triturated with $10 \%$ sodium bicarbonate solution. The neutral solution was poured onto crushed ice and filtered the precipitate of product. The product was dried and crystallized using an appropriate solvent (Scheme 1).

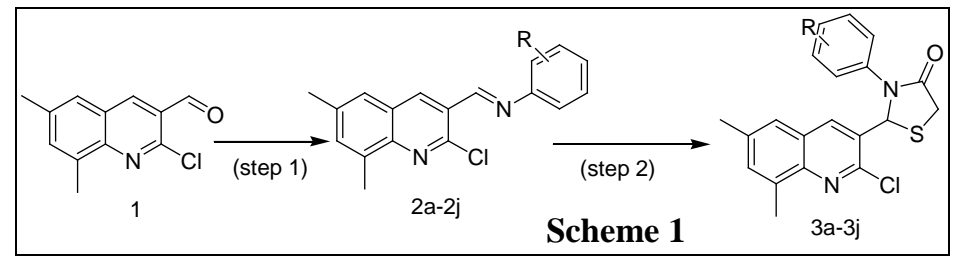

Step 1: $\mathrm{R}_{-} \mathrm{C}_{6} \mathrm{H}_{4}-\mathrm{NH}_{2}$, cat. $\mathrm{CH}_{3} \mathrm{COOH}$, methanol, refulx, 18 h, Step 2: $\mathrm{SHCH}_{2} \mathrm{COOH}, 1,4-$ dioxan, reflux, $18 \mathrm{~h}, \mathrm{R}=$ different substitution 


\section{Antimicrobial activity}

The antimicrobial activity was assay by using the disc diffusion method. Newly synthesized compounds were screened in vitro for their antimicrobial activity against four bacterial strains, i.e. two gram +ve baceteria Staphylococcus epidermidis and Staphylococcus aureus and two gram -ve bacteria Escherichia coli and Pseudomonas aeruginosa and fungi strain Aspergillus niger. Standard drug Cephalexin and Greseofulvin were used for the comparison purpose. The obtained results for compounds $\mathbf{3 a - 3 \mathbf { j }}$ are recorded Table 2 .

Table 2. Antimicrobial screening results of 1-acetyl-4-[5-(3-chloro-4-fluorophenyl)-2furyl]-3-substituted phenyl-4,5-dihydro- $1 H$-pyrazoles (3a-3j)

\begin{tabular}{|c|c|c|c|c|c|c|}
\hline \multirow{3}{*}{ Compd No. } & \multirow{3}{*}{$\mathrm{R}$} & \multicolumn{5}{|c|}{ Zone of inhibition, in mm } \\
\hline & & \multicolumn{4}{|c|}{ Antibacterial activity } & \multirow{2}{*}{$\begin{array}{c}\begin{array}{c}\text { Antifungal } \\
\text { Activity, \% }\end{array} \\
\text { A.niger } \\
\end{array}$} \\
\hline & & S. aureus & S.epidermiss & E.coli & P.aeruginosa & \\
\hline 3a & $\mathrm{C}_{6} \mathrm{H}_{5-}^{-}$ & 72 & 41 & 32 & 71 & 60 \\
\hline $3 \mathbf{b}$ & $4-\mathrm{Br} \mathrm{C}_{6} \mathrm{H}_{4^{-}}$ & 54 & 69 & 89 & 84 & 72 \\
\hline $3 c$ & $4-\mathrm{Cl}-\mathrm{C}_{6} \mathrm{H}_{4}^{-}$ & 77 & 37 & 81 & 77 & 78 \\
\hline 3d & 2,4-di Cl- $\mathrm{C}_{6} \mathrm{H}_{3}-$ & 80 & 85 & 72 & 82 & 83 \\
\hline $3 \mathbf{e}$ & $4-\mathrm{F}-\mathrm{C}_{6} \mathrm{H}_{4}-$ & 58 & 78 & 63 & 86 & 63 \\
\hline $3 f$ & $4-\mathrm{CH}_{3}-\mathrm{C}_{6} \mathrm{H}_{4}^{-}$ & 71 & 59 & 74 & 68 & 67 \\
\hline $3 g$ & $4-\mathrm{NO}_{2}-\mathrm{C}_{6} \mathrm{H}_{4^{-}}$ & 70 & 71 & 89 & 72 & 70 \\
\hline $3 \mathbf{h}$ & $4-\mathrm{SCH}_{3}-\mathrm{C}_{6} \mathrm{H}_{4-}$ & 69 & 47 & 61 & 66 & 63 \\
\hline $3 \mathbf{i}$ & $4-\mathrm{OCH}_{3}-\mathrm{C}_{6} \mathrm{H}_{4^{-}}$ & 50 & 54 & 79 & 61 & 68 \\
\hline $3 \mathbf{j}$ & $4-\mathrm{CH}_{3}-\mathrm{C}_{6} \mathrm{H}_{4}^{-}$ & 81 & 68 & 50 & 39 & 64 \\
\hline Cephalexin & - & 100 & 100 & 100 & 100 & - \\
\hline Greseofulvin & - & - & - & - & - & 100 \\
\hline
\end{tabular}

\section{Spectroscopic data}

2-(2-Chloro-6,8-dimethylquinolin-3-yl)-3-phenyl-1,3-thiazolidin-4-one(3a)

${ }^{1} \mathrm{H}$ NMR (DMSO-d ${ }_{6}$ ) $\delta p p m: 2.42\left(3 \mathrm{H}, \mathrm{s}, \mathrm{Ar}-\mathrm{CH}_{3}\right), 2.44\left(3 \mathrm{H}, \mathrm{s}, \mathrm{Ar}-\mathrm{CH}_{3}\right), 3.90(1 \mathrm{H}, \mathrm{d},-\mathrm{CHa})$, 4.10(1H,d, -CHb), 6.70(1H,s,-CH), $7.11(1 \mathrm{H}, \mathrm{s}, \mathrm{Ar}-\mathrm{H}), 7.20-7.70(5 \mathrm{H}, \mathrm{m}, \mathrm{Ar}-\mathrm{H}), 7.8$ (1H,s,Ar-H), 7.9(1H,d, Ar-H) ; IR ( $\left.\mathrm{cm}^{-1} \mathrm{KBr}\right) 1733(\mathrm{C}=\mathrm{O}$, thiazolidinone), 1608(C=N Str.), 1518 (C=C Str),781(C-S-C), 750 (C-Cl).

3-(4-Bromophenyl)-2-(2-chloro-6,8-dimethylquinolin-3-yl)-1,3-thiazolidin-4-one(3b)

${ }^{1} \mathrm{H}$ NMR (DMSO-d $\left.{ }_{6}\right) \delta \mathrm{ppm}: 2.44\left(3 \mathrm{H}, \mathrm{s}, \mathrm{Ar}-\mathrm{CH}_{3}\right), 2.46\left(3 \mathrm{H}, \mathrm{s}, \mathrm{Ar}-\mathrm{CH}_{3}\right), 3.92(1 \mathrm{H}, \mathrm{d},-\mathrm{CHa})$, 4.11(1H,d, -CHb), 6.68(1H,s,-CH), 7.15 (1H,s, Ar-H), 7.42(2H,d, Ar-H), 7.46(2H,d,Ar-H), 7.82(1H,s,Ar-H), 7.90(1H,d, Ar-H); IR $\left(v^{-1} \mathrm{~cm}^{-1} \mathrm{KBr}\right) 1733(\mathrm{C}=\mathrm{O}$, thiazolidinone $), 1608(\mathrm{C}=\mathrm{N}$ Str.), 1518 (C=C Str),781(C-S-C), 750 (C-Cl), 749(C-Br).

2-(2-Chloro-6,8-dimethylquinolin-3-yl)-3-(4-chlorophenyl)-1,3-thiazolidin-4-one (3c) ${ }^{1} \mathrm{H}$ NMR (DMSO-d $\left.{ }_{6}\right) \delta \mathrm{ppm}: 2.41\left(3 \mathrm{H}, \mathrm{s}, \mathrm{Ar}-\mathrm{CH}_{3}\right), 2.43\left(3 \mathrm{H}, \mathrm{s}, \mathrm{Ar}-\mathrm{CH}_{3}\right), 3.94(1 \mathrm{H}, \mathrm{d},-\mathrm{CHa})$, 4.13(1H,d, -CHb), 6.65(1H,s,-CH), 7.13(1H,s, Ar-H), 7.16(2H,d, Ar-H), 7.62(2H,d,Ar-H), 7.84(1H,s,Ar-H), 7.90(1H,d, Ar-H); IR ( $\left.v \mathrm{~cm}^{-1} \mathrm{KBr}\right) 1733(\mathrm{C}=\mathrm{O}$, thiazolidinone), $1608(\mathrm{C}=\mathrm{N}$ Str.), 1518 (C=C Str),781(C-S-C), 750 (C-Cl). 
2-(2-Chloro-6,8-dimethylquinolin-3-yl)-3-(2,4-dichlorophenyl)-1,3-thiazolidin-4one (3d)

${ }^{1} \mathrm{H}$ NMR (DMSO-d $\left.{ }_{6}\right) \delta p p m: 2.42\left(3 \mathrm{H}, \mathrm{s}, \mathrm{Ar}-\mathrm{CH}_{3}\right), 2.44\left(3 \mathrm{H}, \mathrm{s}, \mathrm{Ar}-\mathrm{CH}_{3}\right), 3.82(1 \mathrm{H}, \mathrm{d},-\mathrm{CHa})$, 3.90(1H,d, $-\mathrm{CHb}), 6.90(1 \mathrm{H}, \mathrm{s},-\mathrm{CH}), 7.20-8.30(6 \mathrm{H}, \mathrm{m}, \mathrm{Ar}-\mathrm{H})$; IR $\left(\mathrm{v} \mathrm{cm}^{-1} \mathrm{KBr}\right) 1733(\mathrm{C}=\mathrm{O}$, thiazolidinone), 1608(C=N Str.), 1518 (C=C Str),781(C-S-C), 750 (C-Cl).

2-(2-Chloro-6,8-dimethylquinolin-3-yl)-3-(4-fluorophenyl)-1,3-thiazolidin-4-one (3e) ${ }^{1} \mathrm{H}$ NMR (DMSO-d $\left.{ }_{6}\right) \delta p p m ~: ~ 2.44\left(3 \mathrm{H}, \mathrm{s}, \mathrm{Ar}-\mathrm{CH}_{3}\right), 2.47\left(3 \mathrm{H}, \mathrm{s}, \mathrm{Ar}-\mathrm{CH}_{3}\right), 3.86(1 \mathrm{H}, \mathrm{d},-\mathrm{CHa})$, 3.88(1H,d, -CHb), 6.80(1H,s,-CH), 7.11 (1H,s, Ar-H), 7.12(2H,d, Ar-H), 7.88(2H,d,Ar-H), 7.80(1H,s,Ar-H), 7.90(1H,d, Ar-H); IR ( $\left.\mathrm{v} \mathrm{cm}^{-1} \mathrm{KBr}\right) 1733(\mathrm{C}=\mathrm{O}$, thiazolidinone), 1608(C=N Str.), 1518 (C=C Str),781(C-S-C), 750 (C-Cl), 680(C-F).

2-(2-Chloro-6,8-dimethylquinolin-3-yl)-3-(4-nitrophenyl)-1,3-thiazolidin-4-one (3f) ${ }^{1} \mathrm{H}$ NMR (DMSO-d $\left.{ }_{6}\right) \delta p p m: 2.42\left(3 \mathrm{H}, \mathrm{s}, \mathrm{Ar}-\mathrm{CH}_{3}\right), 2.44\left(3 \mathrm{H}, \mathrm{s}, \mathrm{Ar}-\mathrm{CH}_{3}\right), 3.85(1 \mathrm{H}, \mathrm{d},-\mathrm{CHa})$, 3.90(1H,d, $-\mathrm{CHb}), 6.82(1 \mathrm{H}, \mathrm{s},-\mathrm{CH}), 7.11(1 \mathrm{H}, \mathrm{s}, \mathrm{Ar}-\mathrm{H}), 7.66(2 \mathrm{H}, \mathrm{d}, \mathrm{Ar}-\mathrm{H}), 8.22(2 \mathrm{H}, \mathrm{d}, \mathrm{Ar}-\mathrm{H})$, 7.76(1H,d, Ar-H), 7.80(1H,s,Ar-H); IR ( $\left.v \mathrm{~cm}^{-1} \mathrm{KBr}\right) 1733(\mathrm{C}=\mathrm{O}$, thiazolidinone $), 1608(\mathrm{C}=\mathrm{N}$ Str.), 1518 (C=C Str),1105(-NO2), 781(C-S-C), 750 (C-Cl).

2-(2-Chloro-6,8-dimethylquinolin-3-yl)-3-[4-(methylsulfanyl)phenyl]-1,3-thiazolidin 4-one (3g)

${ }^{1} \mathrm{H}$ NMR (DMSO-d $\left.{ }_{6}\right) \delta p p m ~: ~ 2.42\left(3 \mathrm{H}, \mathrm{s}, \mathrm{Ar}-\mathrm{CH}_{3}\right), 2.44\left(3 \mathrm{H}, \mathrm{s}, \mathrm{Ar}-\mathrm{CH}_{3}\right), 2.46(3 \mathrm{H}, \mathrm{s}-\mathrm{SCH} 3)$, 3.82(1H,d, -CHa), 3.88(1H,d, -CHb), 6.82(1H,s,-CH), 7.12(1H,m, Ar-H), 7.38(2H,d, Ar-H), 7.42(2H,d,Ar-H), 7.80(1H,s, Ar-H), 7.88(1H,d,Ar-H); IR $\left(v \mathrm{~cm}^{-1} \mathrm{KBr}\right)$ 1733(C=O, thiazolidinone), 1608(C=N Str.), 1518 (C=C Str),781(C-S-C), 750 (C-Cl), 700(C-S).

2-(2-Chloro-6,8-dimethylquinolin-3-yl)-3-(4-methoxyphenyl)-1,3-thiazolidin-4-one (3h)

${ }^{1} \mathrm{H}$ NMR (DMSO-d $\left.{ }_{6}\right) \delta p p m ~: ~ 2.42\left(3 \mathrm{H}, \mathrm{s}, \mathrm{Ar}-\mathrm{CH}_{3}\right), 2.44\left(3 \mathrm{H}, \mathrm{s}, \mathrm{Ar}-\mathrm{CH}_{3}\right), 3.80(3 \mathrm{H}, \mathrm{s}-\mathrm{OCH} 3)$, 3.84(1H,d, -CHa), 3.90(1H,d, -CHb), 6.66(1H,s,-CH), 7.12(1H,m, Ar-H), 6.80(2H,d, Ar-H), 7.50(2H,d,Ar-H), 7.80(1H,s, Ar-H), 7.95(1H,d,Ar-H); IR $\left(v \mathrm{~cm}^{-1} \mathrm{KBr}\right) 1733(\mathrm{C}=\mathrm{O}$, thiazolidinone), 1608(C=N Str.), 1518 (C=C Str),1200(C-O), 781(C-S-C), 750 (C-Cl).

\section{2-(2-Chloro-6,8-dimethylquinolin-3-yl)-3-phenyl-1,3-thiazolidin-4-one (3i)}

${ }^{1} \mathrm{H}$ NMR (DMSO-d $\left.\mathrm{d}_{6}\right) \delta \mathrm{ppm}: 2.38\left(3 \mathrm{H}, \mathrm{s}, \mathrm{Ar}-\mathrm{CH}_{3}\right), 2.46\left(3 \mathrm{H}, \mathrm{s}, \mathrm{Ar}-\mathrm{CH}_{3}\right), 2.48(3 \mathrm{H}, \mathrm{s}, \mathrm{Ar}-\mathrm{CH} 3)$, 3.84(1H,d, -CHa), 3.87(1H,d, -CHb), 6.70(1H,s,-CH), $7.12(1 \mathrm{H}, \mathrm{m}, \mathrm{Ar}-\mathrm{H}), 7.28(2 \mathrm{H}, \mathrm{d}, \mathrm{Ar}-\mathrm{H})$, 7.56(2H,d,Ar-H), 7.80(1H,s,Ar-H), 7.90(1H,d, Ar-H); IR $\left(v \mathrm{~cm}^{-1} \mathrm{KBr}\right) 1733(\mathrm{C}=\mathrm{O}$, thiazolidinone), 1608(C=N Str.), 1518 (C=C Str),781(C-S-C), 750 (C-Cl).

2-(2-Chloro-6,8-dimethylquinolin-3-yl)-3-(2,4-dimethylphenyl)-1,3-thiazolidin-4-one

(3j)

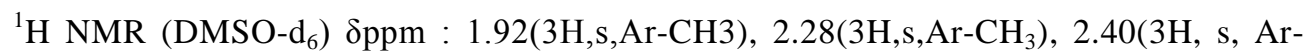
$\left.\mathrm{CH}_{3}\right), \quad 2.44(3 \mathrm{H}, \mathrm{s}, \mathrm{Ar}-\mathrm{CH} 3), \quad 3.86(1 \mathrm{H}, \mathrm{d},-\mathrm{CHa}), \quad 3.90(1 \mathrm{H}, \mathrm{d},-\mathrm{CHb}), 6.66(1 \mathrm{H}, \mathrm{s},-\mathrm{CH})$, 6.88(1H,m,Ar-H), 6.98(1H,m,Ar-H), 7.12 (1H,m, Ar-H), 7.42(1H,m,Ar-H), 7.76(1H,s,Ar-H), 7.83(1H,d, Ar-H); IR ( $\left(\mathrm{cm}^{-1} \mathrm{KBr}\right) 1733(\mathrm{C}=\mathrm{O}$, thiazolidinone $), 1608(\mathrm{C}=\mathrm{N} \mathrm{Str}),$. (C=C Str),781(C-S-C), 750 (C-Cl).

\section{Results and Discussion}

1,3-Thiazolidine-4-on compounds (3a-3j) have been synthesized by the reaction of $N$-[(1E)(3-chloro-5,7-dimethyl-2-naphthyl) methylene]aniline (2a-2j) with thio aceticacid with 60 to 
$80 \%$ of good yield. The structure of $\mathbf{3 a} \mathbf{a}-\mathbf{3} \mathbf{j}$ compounds is confirmed by IR, ${ }^{1} \mathrm{HNMR}$ and mass spectral data analysis. From the results of antimicrobial data, compounds $\mathbf{3 c}$, $\mathbf{3 d}$ and $\mathbf{3 g}$ were showing excellent active against selected gram positive and negative bacterial strains as compare to standard drug cephalexin, while $\mathbf{3 a}, \mathbf{3 b}, \mathbf{3 e}$ and $\mathbf{3 f}$ were moderately active against selected bacterial strains as compare to standard drug. While $\mathbf{3 c}$, $\mathbf{3 d}$ and $\mathbf{3 g}$ were also shown excellent activity aginst fungi strain A.niger as compare to standard drug griseofulvin. From the structure activity relationship table, we find that phenyl ring substituted with chloro (3c) and flouro (3g) at shown an excellent result compare to standard drug cephalexin at a scale of $40 \mathrm{ug} / \mathrm{mL}$.

\section{Conclusion}

From the antimicrobial results, it worthwhile to say that, newly synthesized 1,3-thiazolidine4-one derivatives are showing moderate to good activity against bacterial and fungi strains. From the structure activity relationship table and the results we found that, phenyl rings substituted with chloro and flouro 2,3 and 4 position will show good activity as compare to other functional groups. From the obtained data for the newly synthesized pyrazoline derivatives we conclude on that with presence of halogen substitution in pyrazoline derivative will show excellent antimicrobial activity. And will give us path to synthesize more number of compounds with variations of halogen substitution on different ring, which may hope to give a better antimicrobial compound in the true sense.

\section{Acknowledgement}

Authors are thankful to Department of Chemistry, Navjivan Science College, Dahod for providing facility.

\section{References}

1. Abdou W M, Khidre R E and Azza A. Kamel, Arch Pharm Chem Life Sci., 2012, 345, $123-136$.

2. Charris J E, Lobo E G, Camacho J, Ferrer R, Barazarte A, Dominguez J N, Gamboa N, Rodrigues J R and Angel J E, Lett Drug Design Discov., 2007, 4, 49-54.

3. Yeh-Long Chen Kuo-Chang Fang Jia-Yuh Sheu, Shu-Lin Hsu and Cherng-Chyi Tzeng, J Med Chem., 2001, 44(14), 2374-2377.

4. Lokhandwala S R and Desai K R, Phosphorus Sulfur Silicon Relat Elem., 2008, 183, 1264-1271.

5. Geronikaki A A, Lagunin A A, Hadjipavlou-Litina D I, Eleftheriou P T, Filimonov D A, Poroikov V V, Alam I and Saxena A K, J Med Chem., 2008, 51, 1601-1609.

6. Balzarini J, Orzeszko-Krzesinska B, Maurin J K and Orzeszko A, Eur J Med Chem., 2009, 44, 303-311.

7. Tenorio R P, Carvalho C S, Pessanha C S, de Lima J G, de Faria A R, Alves A J, de Melo E J T and Goes A J S, Bioorg Med Chem Lett., 2005, 15, 2575-2578.

8. Vigorita M G, Ottana R, Monforte F, Maccari R, Trovato A, Monforte M T and Taviano M, Bioorg Med Chem Lett., 2001, 11, 2791-2794. 